\title{
Limits of normality and symmetry in standing back shape and posture
}

\author{
Josette Bettany-Saltikov*, JG Warren, J Robinson \\ From 7th International Conference on Conservative Management of Spinal Deformities \\ Montreal, Canada. 20-22 May 2010
}

\begin{abstract}
Introduction
Abnormalities of posture are a common cause of pain and disability. The range of effect from discomfort to incapacitating disability is related both to the severity as well as to the persistence of the faults (Kendall, 1993). On the whole, objective measurement systems for postural evaluation are not widely accessible in the UK especially on the National Health Service (Warren et al, 2005). Within physiotherapy practice one of the most common methods of assessing posture is by visual observation. It is well known however that unaided visual assessment is prone to error and lacks objectivity. Accurate recording of back shape and posture, if scientifically based on reliable measures can be used to credibly validate intervention or efficacy of treatment. However uncertainly still remains as to the limits of normality relating to back shape values in young males and females. Knowledge of these values would significantly benefit clinicians as it would enable them to decide when postural retraining exercises/ conservative treatment is warranted. The objective of this study was to identify the limits of normality and symmetry of back shape in a group of asymptomatic young females and males.
\end{abstract}

\section{Materials and methods}

A convenience sample of 100 Teesside University students aged 18-40 were recruited for this study. The Microscribe digitiser described in previous studies was used to digitise 15 bony landmarks on the back.

\section{Results}

Young adult male subjects were found to be very symmetrical overall. All mean distances between key anatomical landmarks on the back were larger than for

Teesside University, Middlesbrough, UK

Full list of author information is available at the end of the article female ones as expected. However no significant differences were found between key distances on the left and right sides of the back. In females, the only significant differences between the left and right sides of the back were found in the distance between the acromium and the inferior scapula. The right side was significantly smaller than the left side. Further the distance between the inferior angle of the scapula and the apical thoracic vertebra on the right side was significantly longer than the left side. The left shoulder in females was also significantly higher then the right shoulder. No statistically significant differences at the inferior angle of scapula, iliac crests or PSIS were found in females.

\section{Discussion}

The results of this study suggest that young asymptomatic subjects are very symmetrical with males being more symmetrical than females. In females asymmetries exist predominantly around the shoulder.

\section{Conclusions}

Ranges for normality of back shape and posture suggest that overall young asymptomatic males and females are very symmetrical, with the exception of shoulder values in young females. The normative ranges provided should help clinicians decide when postural retraining exercises/ conservative treatment is warranted as well as aid the documentation of treatment effectiveness.

Published: 10 September 2010

doi:10.1186/1748-7161-5-S1-O5

Cite this article as: Bettany-Saltikov et al:: Limits of normality and symmetry in standing back shape and posture. Scoliosis 2010 5(Suppl 1): O5. 